\title{
ПРОГРАММНО-АППАРАТНЫЙ КОМПЛЕКС ДЛЯ УПРАВЛЕНИЯ БИОТЕХНОЛОГИЧЕСКИМИ СИСТЕМАМИ С ИСПОЛЬЗОВАНИЕМ ИНТЕЛЛЕКТУАЛЬНЫХ ИНФОРМАЦИОННЫХ ТЕХНОЛОГИЙ
}

\author{
С. Г. Тихомиров ${ }^{\star}$, И. А. Авцинов ${ }^{\star}$, Я. А. Туровский ${ }^{\star \star, \star * \star}$, \\ А. С. Суровцев ${ }^{\star}$ А. А. Адаменко ${ }^{\star}$ Е. Н. Ковалева ${ }^{\star}$ \\ *Воронежский государственный университет инженерных технологий \\ ${ }^{*}$ Воронежский государственный университет \\ ***Институт проблем управления им. В. А. Трапезникова РАН
}

\section{Поступила в редакцию 25.06.2019 г.}

\begin{abstract}
Аннотация. Предложен подход к управлению биотехническими системами, который заключается в использовании интеллектуальных информационных технологий в системе их управления. Рассмотрен вариант построения системы управления, в качестве одного из элементов которой выступает искусственная нейронная сеть. Для обучения искусственных нейронных сетей в работе использован эволюционный алгоритм. Проведена оптимизация эволюционного алгоритма обучения искусственных нейронных с использованием механизма «изоляции». Разработан программно-аппаратный комплекс моделирования, идентификации и управления биотехнологической системой. Серией проведенных вычислительных экспериментов показана его работоспособность.

Ключевые слова: эволюционный алгоритм, изоляты, искусственные нейронные сети, аппаратная аналоговая искусственная нейронная сеть, программная искусственная нейронная сеть.
\end{abstract}

\section{ВВЕДЕНИЕ}

Современные биотехнологические системы - это широкий класс систем, которые используются для получения продуктов различного назначения. В работах [1-3] установлено, что при управлении такими системами возникает проблема параметрической идентификации. Авторами статей $[1,2]$ для решения этой проблемы приводятся алгоритмы, в которых используются методы энтропийных потенциалов. В этих статьях с помощью методов энтропийных потенциалов выполняется построение так называемых «информационных портретов» для параметров, оценки значений которых требуется получить. Таким образом, значения части элементов системы описываются оценками, полученными с использованием «информационных портре-

() Тихомиров С. Г., Авцинов И. А., Туровский Я. А., Суровцев А. С., Адаменко А. А., Ковалева Е. Н., 2019 тов». Исходя из этого, авторы предлагают выполнять робастное управление. Показано, что применение таких алгоритмов для решения проблемы параметрической идентификации является достаточным для построения систем управления ряда биотехнологических систем. Однако, для построения систем управления множества биотехнологических систем необходимо учитывать не только состояние системы, но и причинно-следственные связи, привлекшие к изменению состояния этой системы. Поэтому, может иметь место также и структурная неопределенность.

При построении системы управления биотехнологической системой возникают две проблемы - параметрическая и структурная неопределенности. Наличие этих проблем объясняется тем, что большинство биотехнологических систем являются динамическими. Поэтому, одним из способов реализации системы управления биотехнологическими системами является построение её математи- 
Программно-аппаратный комплекс для управления биотехнологическими системами ...

ческой модели. Наличие параметрической и структурной неопределенностей обуславливает сложность получения функции ошибки в явном виде для идентификации параметров математических моделей. Для идентификации параметров математических моделей, когда имеет место сложность задания функции ошибки в явном виде, применяют эволюционные алгоритмы $[4,5]$. В связи с этим, для поддержания нормального режима работы биотехнических систем, в систему их управления может быть включен элемент, который реализует математическую модель, обучающуюся с использованием эволюционного алгоритма.

Авторы работы [6] впервые предложили использовать метод «изоляции» при обучении искусственных нейронных сетей (ИНС) с использованием эволюционного метода. В работах $[5,6]$ показано повышение точности обучения ИНС при использовании метода «изоляции» для обучения ИНС эволюционным методом. Вследствие чего, в качестве математической модели, описывающей поведение биотехнологической системы, можно использовать ИНС, обучаемую эволюционным методом с использованием механизма «изоляции».

Целью данной работы является построение программно-аппаратного комплекса моделирования, идентификации и управления биотехнологическими системами на основе эволюционного алгоритма с использованием механизма «изоляции».

\section{РЕЗУЛЬТАТЫ СИСТЕМНОГО АНАЛИЗА И ИХ ОБСУЖДЕНИЕ}

В результате проведения системного анализа процесса управления обобщенной биотехнологической системы построена структурно-информационная модель её управления (рис. 1). «Аналоговая аппаратная искусственная нейронная сеть» (ААИНС) выступает в качестве модели биотехнологической системы(объект управления) (рис. 1). Субъ-

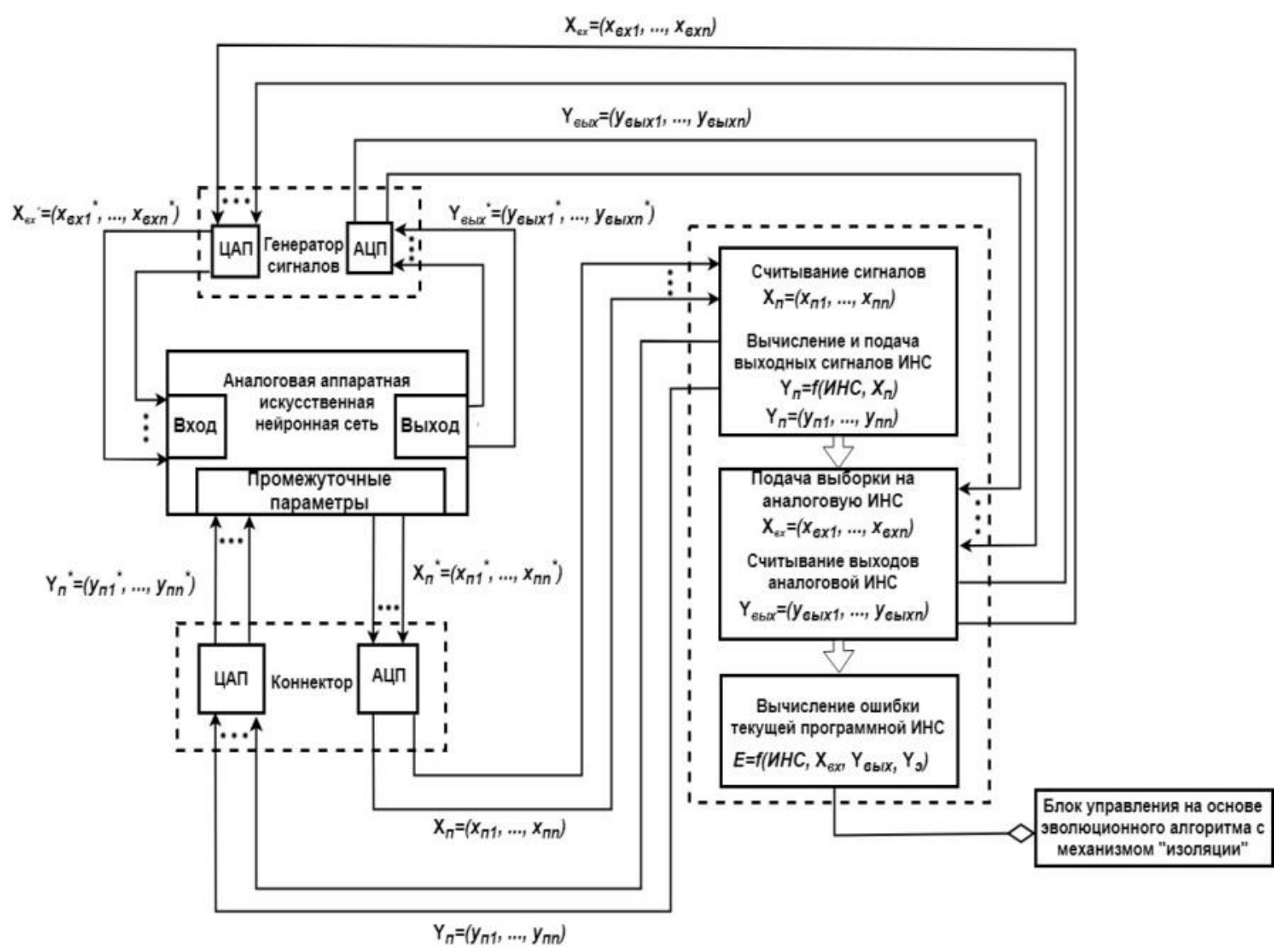

Рис. 1. Структурно-информационная модель управления биотехнологической системой 
С. Г. Тихомиров, И. А. Авцинов, Я. А. Туровский, А. С. Суровцев, А. А. Адаменко, Е. Н. Ковалева

ектом управления данной модели (рис. 1) является «блок управления», в состав которого входит искусственная нейронная сеть (ИНС). Связь ААИНС и «блока управления» осуществляется через «генератор сигналов» и «коннектор».

Большинство биотехнологических систем являются динамическими. Поэтому субъект управления такими системами нередко может требовать адаптации после изменений в объекте управления. В данной структурной- информационной модели адаптация заключается в обучении ИНС «объекта управления». Для обучения ИНС используется эволюционный алгоритм $[4,5]$. На первом этапе эволюционного алгоритма генерируется множество сетей (нулевая популяция или $\{A N N\}^{0}$ ) со случайно выбранными весовыми коэффициентами (ВК). Скрещивание ИНС для формирования следующих популяций $\{A N N\}^{p}$ происходит следующим образом. Выбираются множество пар из текущей популяции. Каждая из выбранных пар пара $A^{p}, B^{p} \in\{A N N\}^{p}$ подвергается скрещиванию. В результате скрещивания образуется одна нейронная сеть, ВК которой вычисляются по формуле (1):

$$
c_{\left\{l_{j}\right\},\left\{n_{j}\right\},\left\{w_{k}\right\}}^{p+1}=\frac{a_{\left\{l_{j}\right\},\left\{n_{j}\right\},\left\{w_{k}\right\}}^{p}+b_{\left\{l_{j}\right\},\left\{n_{j}\right\},\left\{w_{k}\right\}}^{p}}{2},
$$

где $i$ - номер слоя из множества слоев $\{l\} ; j-$ номер нейрона из множества нейронов $\{n\} \in\left\{l_{j}\right\} ; k$ - номер веса из множества весов $\{w\} \in\left\{n_{j}\right\} ; p$ - номер текущего поколения; $A$ и $B$ случайно выбранные сети из текущего поколения и прошедшие отбрасывание наихудших сетей; $C$ - сеть для следующего поколения.

Множество пар, полученных после скрещивания пар ИНС, выбранных из текущей популяции, подвергаются мутации:

$$
\begin{aligned}
& A_{\left\{l_{j}\right\},\left\{n_{j}\right\},\left\{w_{k}\right\}}^{p}=A_{\left\{l_{j}\right\},\left\{n_{j}\right\},\left\{w_{k}\right\}}^{p} \times \\
& \times \operatorname{RandomSign}\left(A_{\left\{l_{j}\right\},\left\{n_{j}\right\},\left\{w_{k}\right\}}^{p}\right),
\end{aligned}
$$

где $A^{p}$ - сеть поколения $p$ показавшая наихудший результат из всех сетей поколения; $i$ - номер слоя из множества слоев $\{l\} ; j-$ номер нейрона из множества нейронов $\{n\} \in\left\{l_{j}\right\} ; k-$ номер веса из множества весов $\{w\} \in\left\{n_{j}\right\}$, с коэффициентом вариации ниже заданного; RandomSign - случайный знак выражения: «+», «-».

\section{ОПТИМИЗАЦИЯ ЭВОЛЮЦИОННОГО АЛГОРИТМА ОБУЧЕНИЯ ИСКУССТВЕННЫХ НЕЙРОННЫХ СЕТЕЙ НА ОСНОВЕ МЕХАНИЗМА «ИЗОЛЯЦИИ»}

Разработан механизм «изоляции», который призван улучшить эволюционный метод обучения ИНС [6]. Механизм «изоляции» предлагает формировать набор изолятов. «Изолят» - множество ИНС одного поколения, которые имеют одну и ту же родительскую нейронную сеть. Формирование ВК $w_{i}^{\partial}$ дочерней ИНС на основе ВК $w_{i}^{p}$ родительской ИНС представлено в следующей формуле:

Набор сетей, которые образуют «изолят», получается изменением ВК клонов родительской ИНС в пределах некоторого заданного диапазона значений. Имея набор дочерних ИНС, ВК которых в $n$-мерном пространстве лежат внутри некоторой окрестности родительской ИНС, есть возможность определить направление движение в $n$-мерном пространстве ВК для достижения локального минимума функции ошибки.

Каждый $i$-й ВК $w_{i}^{d}$ в дочерней ИНС изменяется по отношению к родительскому ВК $w_{i}^{p}$ следующим образом:

$$
w_{i}^{\partial}=w_{i}^{p}\left(1+\frac{r}{q}\right)
$$

где $r \in[-1 ; 1]$ является случайной величиной; $q$ - заданный процент изменения ВК дочерней ИНС; $i=1 \ldots n$, где $n-$ количество всех ВК ИНС.

Вектор $W$ ВК всей ИНС можно представить в виде совокупности ее ВК:

$$
W=\left(w_{1}, w_{2}, \ldots, w_{n}\right) .
$$

Путем изменения родительских векторов создается $k$ дочерних ИНС, содержащие векторы ВК вида (2) от родительской ИНС:

$$
W^{\partial \kappa}=\left(w_{1}^{\partial \kappa}, w_{2}^{\partial \kappa}, \ldots, w_{n}^{\partial \kappa}\right),
$$

где $m$ - количество ИНС в поколении.

Из $t$ «изолятов» выбираются ИНС, позволяющие получить более точные результаты моделирования, для формирования множества родительских ИНС.

$$
W^{\partial \kappa t}=\left(w_{1}^{\partial \kappa t}, w_{2}^{\partial \kappa t}, \ldots, w_{n}^{\partial \kappa t}\right), \quad t=1 . . l,
$$

где $l$ - максимальное число родительских ИНС, формирующих совокупность «изолятов». 
Программно-аппаратный комплекс для управления биотехнологическими системами ...

\section{ПРОГРАММНЫЙ ПАКЕТ ДЛЯ МОДЕЛИРОВАНИЯ, ИДЕНТИФИКАЦИИ И УПРАВЛЕНИЯ БИОТЕХНОЛОГИЧЕСКОЙ СИСТЕМОЙ}

Для апробации предложенного механизма «изоляции» для обучения ИНС методом эволюционного алгоритма разработан программный пакет $[7,8]$. Данный программный пакет используется для решения задач моделирования, идентификации и управления биотехнологической системой.

Пакет прикладных программ представлен тремя группами модулей:

- создания и обучения ИНС;

- анализа и изменения ИНС;

- процесса корректировки и управления ИНС.

На рис. 2 показана схема взаимодействия модулей разработанного программного комплекса при моделировании создания и адап- тации «блока управления» системы управления биотехнологической системой.

Первым этапом процесса, показанного на рис. 2 , является создание модели нормальной работы биотехнологической системы. Обозначим ИНС, которая обучена для моделирования нормальной работы биотехнологической системы, как ИНС-А. Ошибка обучения ИНС-А вычисляется по следующей формуле:

$$
\text { learningerror }=\frac{\frac{1}{2} * \sum_{i=1}^{n}\left(x_{i}-x_{i}^{\prime}\right)^{2}}{i} * 100,
$$

где $i$ - количество входящих векторов; $x_{i}^{\prime}-$ желаемый результат $i$-го входящего вектора ИНС; $x_{i}$ - полученный результат ИНС для $i$-го входящего вектора.

Процент правильных ответов сети ИНС-А (т. е. процент ошибок) оценивается следующим выражением:

$$
\text { testerror }=\frac{\sum_{i=0}^{n} e_{i}}{i} * 100,
$$

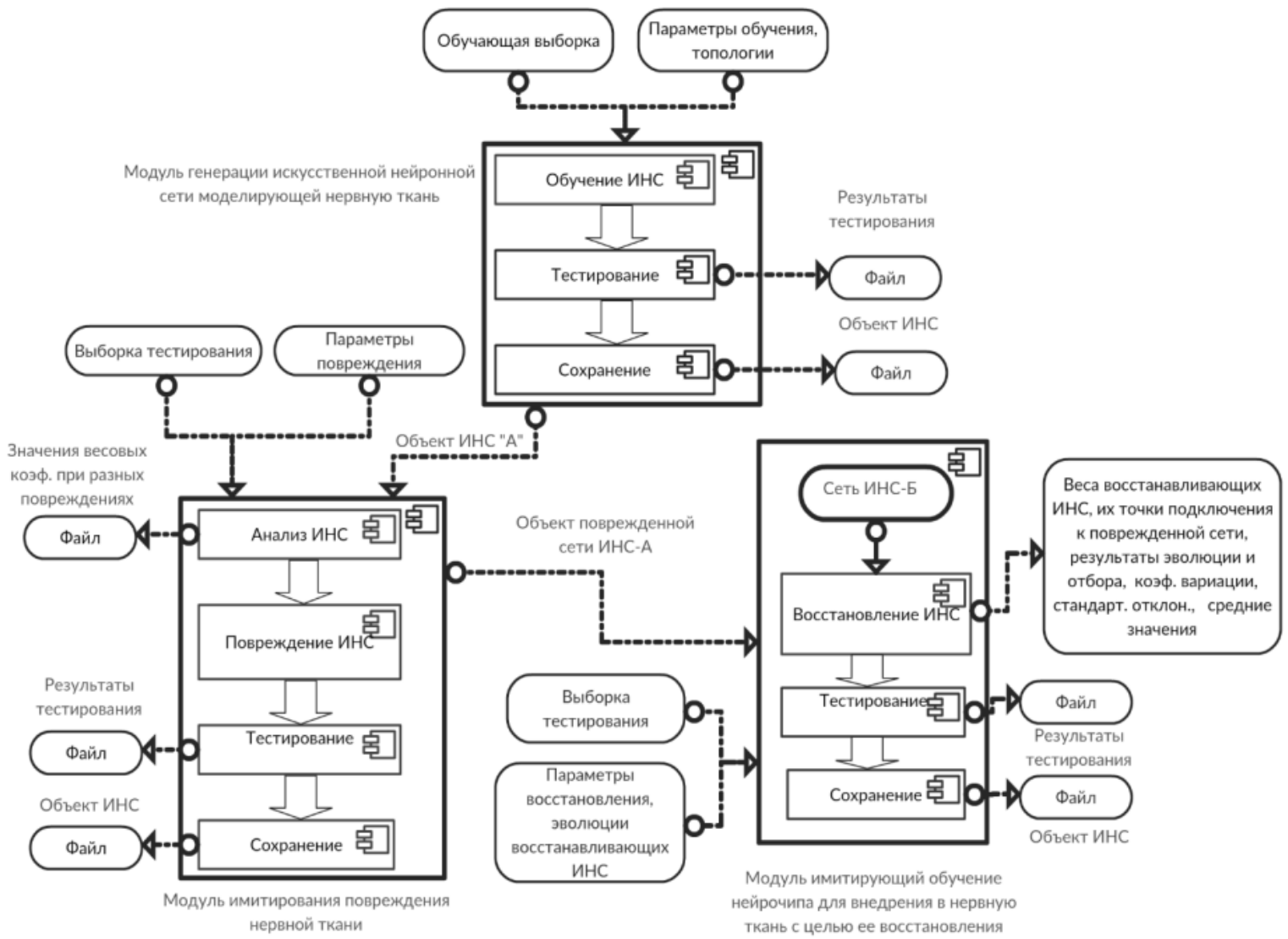

Рис. 2. Схема взаимодействия программных модулей при моделировании процесса обучения нейрочипа или ПИНС для идентификации и управления биотехнологической системой: объект ИНС «А»- обученная нейронная сеть; объект поврежденной сети «ИНС-А»поврежденная нейронная сеть; «Сеть ИНС-Б» - корректировочная нейронная сеть 
С. Г. Тихомиров, И. А. Авичинов, Я. А. Туровский, А. С. Суровиев, А. А. Адаменко, Е. Н. Ковалева

где $i$ - количество входящих векторов; $e_{i}-$ ошибка распознавания для $i$-го входящего вектора сети ИНС-А.

Коэффициент обучения определяется согласно [9]:

$$
w_{p-q}(i+1)=w_{p-q}(i)+\vartheta \delta_{q} O U T_{p},
$$

где $i$ - номер текущей итерации; $w_{p-q}-$ величина синаптического веса, соединяющего нейрон $p$ с нейроном $q ; \vartheta$ - коэффициент «скорости обучения», управляет величиной изменения весов; $\delta_{q}$ - ошибка $q$-го нейрона; $O U T_{p}$ - выход нейрона $p$.

Коррекция весов при обучении с моментом m согласно [10] определяется следующим выражением:

$$
\Delta w_{i j}^{(n)}(t)=-\vartheta * \delta_{j}^{(n)} * x_{i}^{n}+\mu \Delta w_{i j}^{(n)}(t-1),
$$

где $\mu$ - моментный параметр.

Сигмоидальная униполярная функция совместно с параметром альфа реализована следующим образом $[10,11]$ :

$$
f(x)=\frac{1}{1+e^{-x^{*} \alpha}},
$$

где $\alpha$ - альфа параметр наклона. Выбор параметра наклона функций активации может оказывать влияние на диапазоны значений ВК ИНС или выходов нейронов. Это необходимо учитывать при рассмотрении ошибок выполнения операций с плавающей точкой.

На втором этапе происходит изменение ИНС-А, что моделирует трансформацию биотехнологической системы во времени, то есть её отклонение от изначального нормального процесса работы. На данном этапе выполняется тестирование ИНС-А и сохранение результатов в базе данных.

На третьем этапе выполняется корректировка ИНС-А. Создается ИНС-Б, которая моделирует блок управления. ИНС-Б подключается к ИНС-А с целью изменения значений ВК ИНС-А для уменьшения уровня ошибок.

В результате экспериментов по обучению, идентификации и управлению ИНС подтверждена способность разработанного программного пакета моделировать:

- биотехнологическую систему в нормальном режиме работы;

- биотехнологическую систему с отклонениями от нормального режима работы;
- управление биотехнологической системой с использованием эволюционного алгоритма и механизма «изоляции».

\section{РАЗРАБОТКА ПРОГРАММНО- АППАРАТНОГО КОМПЛЕКСА ДЛЯ УПРАВЛЕНИЯ БИОТЕХНОЛОГИЧЕСКИМИ СИСТЕМАМИ}

Для проверки работоспособности предложенного подхода к управлению биотехнологической системой, разработаны «программная искусственная нейронная сеть» (ПИНС) и «аппаратная аналоговая искусственная нейронная сеть» (ААИНС). ПИНС выступает в качестве программной модели биотехнологической системы, а ААИНС аппаратной. ПИНС реализована на языке программирования С\#.

Как говорилось ранее, для связи «блока управления» и объекта управления (в качестве объекта управления могут выступать ПИНС, ААИНС или биотехнологическая система) предложено использовать «генератор сигналов» и «коннектор» (рис. 1). Для аппаратной реализации «генератора сигналов» и «коннектора» использовались Arduino Uno (плата для разработки программно-аппаратных решений с микроконтроллером ATmega328P) [12] и 12-битные ЦАП МСР4921 [13]. Схема соединения ААИНС, «генератора сигналов» и «коннектора» показана на рис. 3.

Проведено 12 вычислительных экспериментов, в рамках которых проводилась оценка поддержания ААИНС в нормальном режиме работы (поддержания эффективности работы) при трансформации ААИНС. Поддержание эффективности работы осуществляется путем адаптации «блока управления». Процесс адаптации «блока управления» заключается в обучении ИНС, которая входит в состав «блока управления», эволюционным подходом с предложенным механизмом «изоляции». Модель биотехнологической системы в данном эксперименте - многослойный персептрон с пятью нейронами в скрытом слое, двумя входами и двумя нейронами на выходе (рис. 3). Имитация трансформации биотехно- 


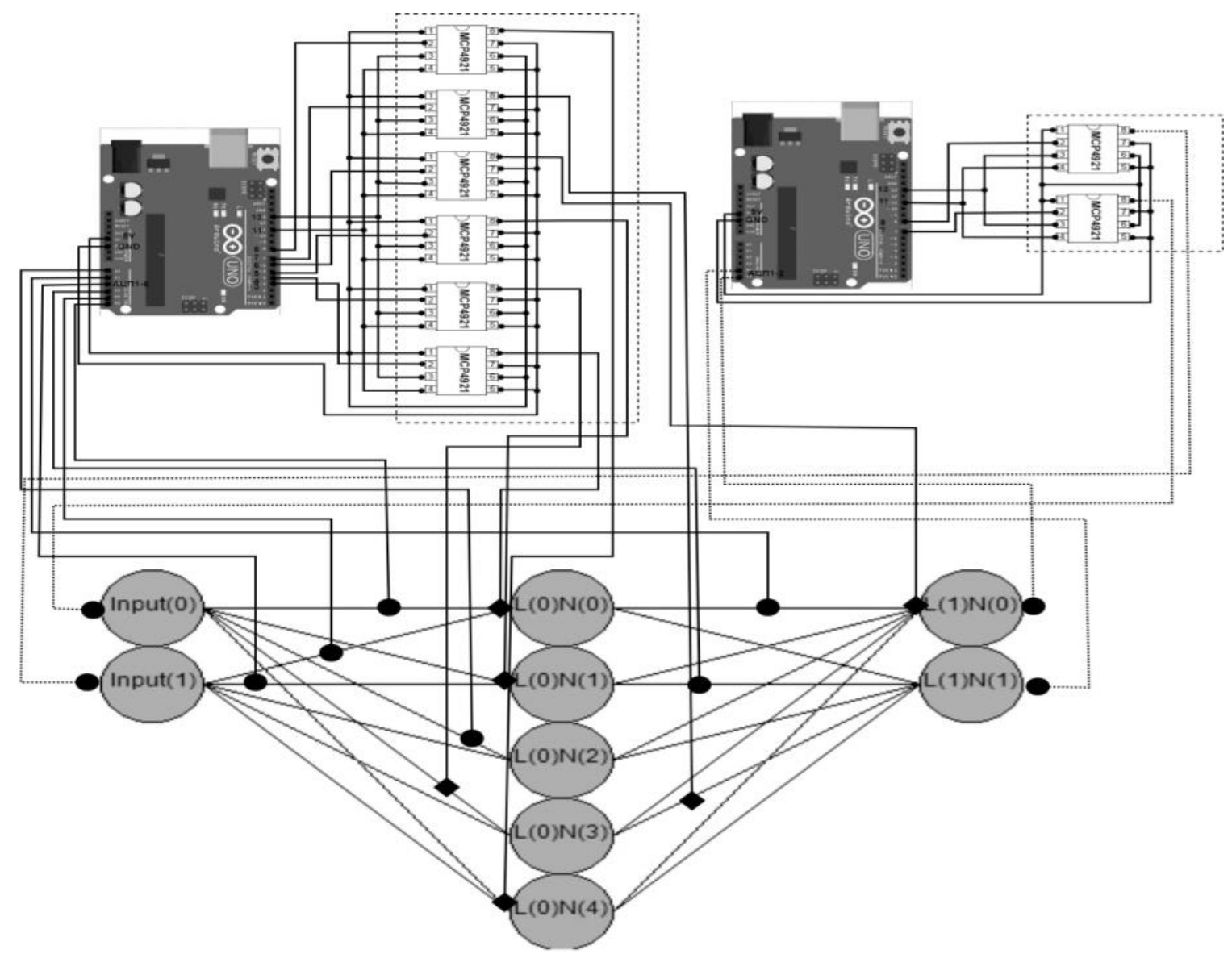

Рис. 3. Общая схема ААИНС с генератором сигналов и коннектором при моделировании идентификации и управления биотехнологической системой

логической системы проводилась путем разъединения контактов ВК ААИНС.

В большинстве случаев удалось восстановить эффективность работы ААИНС до $100 \%$. Лишь при отключении $60 \%$ связей ААИНС (12 связей из 20), эффективность работы была восстановлена с $32 \%$ до 80-95\%. В связи с чем можно утверждать о работоспособности предложенного способа идентификации и управления биотехнологическими системами.

\section{ЗАКЛЮЧЕНИЕ}

Построена структурно-информационная модель управления биотехнологической системой. Разработан программно-аппаратный комплекс, в задачи которого входят идентификация, моделирование и управление биотехнологическими системами. Проведено исследование предложенного подхода управления для поддержания ААИНС в нормальном режиме работы при трансформации ААИНС. Результаты исследования показали высокую эффективность от использования данного подхода управления, поэтому он может быть использован для таких областей как: самообучающиеся и само адаптирующиеся системы; принятие решений и управление, реверс-инжиниринг; восстановление поврежденных банков данных, восстановление изображений; нейропротезирование и т. п.

\section{СПИСОК ЛИТЕРАТУРЫ}

1. Лазарев, В. Л. Совершенствование управления с использованием характеристик энтропийных потенциалов. Адаптация к специфике биотехнологической промышленности // Вестник МАХ. - 2016. - Вып. 4.- С. 68-73.

2. Лазарев, В. Л. Перспективные направления организации робастного управления в биотехнологической промышленности / В. Л. Лазарев, В. Л. Иванов, Н. А. Фролков // 
Низкотемпературные и пищевые технологии в XXI веке. - 2015. - Вып. 1. - С. 279-282.

3. Андрейченко, Д. К. Обучение нейросетевых регуляторов для стабилизации комбинированных динамических систем / Д. К. Андрейченко, Ф. М. Жадаев // Известия Саратовского государственного университета. 2018. - Т. 18, Вып. 3. - С. 354-360.

4. Туровскици, Я. А. Сравнительный анализ эволюционного метода с использованием «изолятов» и метода имитации отжига при обучении искусственных нейронных сетей / Я. А. Туровский, А. А. Адаменко // Программная инженерия. - 2018. - Т. 9, Вып. 4. - С. 185190.

5. Туровский, Я. А. Сравнительный анализ результатов обучения искусственных нейронных сетей в задачах обработки сигналов на основе эволюционного алгоритма с применением и без применения «изоляции» / Я. А. Туровский, А. А. Адаменко // 20-я международная конференция «Цифровая обработка сигналов и ее применение» (DSPA-2018). - 28-30 марта 2018, Москва. - 2018. - С. 241-244.

6. Теоретические аспекты оптимизации эволюционного обучения нейрочипов с использованием «изолятов» / С. Д. Кургалин, Я. А. Туровский, С. В. Борзунов, А. А. Адаменко // Информационные технологии. - 2016. Т. 22, Вып. 11. - С. 888-889.

7. ANNBuilder 2.1.0. - Св-во о гос. peгистрации программы для ЭВМ / Я. А. Ту- ровский, С. Д. Кургалин, А. А. Адаменко. М. : ФИПС, 2015. - № 2016619398; опубл. 18.08.2016. - ФИПС, 2016. - 1 с.

8. Туровский, Я. А. Моделирование обучения нейрочипов, внедренных в нервную ткань / Я. А. Туровский, С. Д. Кургалин, А. А. Адаменко // Цифровая обработка сигналов. - 2016. - С. 13-14.

9. Коэффициент обучения [Электронный pecypc]. - Режим доступа: https://www4.rgu. ac.uk/files/chapter3\%20-\%20bp.pdf. - (Дата обращения: 07.04.2019).

10. Qian, N. On the momentum term in gradient descent learning algorithms / N. Qian // Neural Networks. - 1999. - № 12. - P. 145-151.

11. Функция активации [Электронный ресурс]. - Режим доступа: https://en.wikipedia. org/wiki/Activation_function. - (Дата обращения: 07.04.2019).

12. Функция активации в нейронных сетях [Электронный ресурс]. - Режим доступа: http://www.aiportal.ru/articles/neural-networks/ activation-function.html. - (Дата обращения: 07.04.2019).

13. Arduino Uno [Электронный ресурс]. Режим доступа: https://store.arduino.cc/ usa/arduino-uno-rev3. - (Дата обращения: 07.04.2019).

14. МСР4921 [Электронный ресурс]. Режим доступа: http://www.microchip.com/ wwwproducts/en/MCP4921. - (Дата обращения: 07.04.2019).

Тихомиров Сергей Германович - д-р техн. наук, профессор, профессор кафедры информационных и управляющих систем, ФГБОУВО «Воронежский государственный университет инженерных технологий» (ВГУИТ), e-mail: tikhomirov_57@mail.ru

Авцинов Игорь Алексеевич - д-р техн. наук, профессор, профессор кафедры информационных и управляющих систем, ФГБОУВО «Воронежский государственный университет инженерных технологий» (ВГУИТ), e-mail: igor.awtzinov@yandex.ru

Туровский Ярослав Александрович - канд. мед. наук, доцент, доцент кафедры цифровых технологий, ФГБОУ ВО «Воронежский государственный университет» (ВГУ); старший научный сотрудник института проблем управления им. В. А. Трапезникова РАН (ИПУ РАН), e-mail: yaroslav_turovsk@mail.ru

Суровцев Александр Сергеевич - аспирант кафедры информационных и управляющих систем, ФГБОУ ВО «Воронежский государственный университет инженерных технологий» (ВГУИТ), e-mail: alexander.surovtsev.vrn@gmail.com 
Программно-аппаратный комплекс для управления биотехнологическими системами ...

Адаменко Артем Александрович - аспирант кафедры информационных и управляющих систем, ФГБОУ ВО «Воронежский государственный университет инженерных технологий» (ВГУИТ), e-mail: adamenko.artem@gmail.com

Ковалева Елена Николаевна - канд. техн. наук, доцент, доцент кафедры высшей математики и информационных технологий, ФГБОУ ВО «Воронежский государственный университет инженерных технологий» (ВГУИТ), e-mail: vkovaleva.lena@gmail.com

\title{
HARDWARE-SOFTWARE COMPLEX FOR CONTROL BIOTECHNOLOGICAL SYSTEMS USING INTELLIGENT INFORMATION TECHNOLOGIES
}

\author{
S. G. Tihomirov*, I. A. Awtzinov*, Ya. A. Turovskiy ${ }^{\star \star * \star * *}$, \\ A. S. Surovtsev ${ }^{\star}$ A. А. Адаменко ${ }^{\star}$, А. A. Adamenko* \\ ${ }^{*}$ Voronezh State University of Engineering Technologies \\ ${ }^{*}$ Voronezh State University \\ ${ }^{* * *}$ Institute if Control Sciences RAS
}

\begin{abstract}
Annotation. It is proposed an approach to controlling of biotechnical systems, which consists in using intelligent information technologies in the control system. A variant of constructing the controlling subject is considered, one of an element of which is an artificial neural network. An evolutionary algorithm is used to train artificial neural networks. It is carried out an optimization of the evolutionary algorithm of the training of artificial neural networks by the using of "isolation" mechanism. It is developed software and hardware complex of modelling, identification and control of biotechnical systems. A series of computational experiments shows the efficiency of the proposed method to ensure optimal control of biotechnical systems.

Keywords: evolutionary algorithm, isolates, artificial neural networks, hardware analog artificial neural network, software artificial neural network.
\end{abstract}

Tihomirov Sergey Germanovich - doctor of technical sciences, professor, Voronezh State University of Engineering Technologies, Department of Information and Control Systems, e-mail: tikhomirov_57@mail.ru

Awtzinov Igor Alekseevich - doctor of technical sciences, professor, Voronezh State University of Engineering Technologies, Department of Information and Control Systems, e-mail: igor.awtzinov@ yandex.ru

Turovskiy Yaroslav Aleksandrovich - PhD, Voronezh State University, Department of Digital Technologies; senior researcher, Institute if Control Sciences RAS, e-mail: yaroslav_turovsk@mail.ru

Surovtsev Alexander Sergeevich - Postgraduate Student, Voronezh State University of Engineering Technologies, Department of Information and Control Systems, e-mail: alexander.surovtsev.vrn@ gmail.com

Adamenko Artem Aleksandrovich - Postgraduate Student, Voronezh State University of Engineering Technologies, Department of Information and Control Systems, e-mail: adamenko.artem@gmail. com

Kovaleva Elena Nikolaevna - PhD, Voronezh State University of Engineering Technologies, Department of Higher Mathematics and Information Technologies, e-mail: vkovaleva.lena@gmail.com 